\title{
Design Methodology and Parametric Design Study of the On-Board Electrical Power System for Hybrid Electric Aircraft Propulsion
}

\author{
Giorgio Valente*, Sharmila Sumsurooah**, Christopher Ian Hill, Mohamed Rashed, \\ Gaurang Vakil, Serhiy Bozhko, Chris Gerada \\ Department of Electrical and Electronic Engineering, The University of Nottingham, University Park, \\ Nottingham, $U K$ \\ *giorgio.valente1@nottingham.ac.uk,**sharmila.sumsurooah@nottingham.ac.uk
}

Keywords: Hybrid Electric Aircraft Propulsion, Multi-Disciplinary Analysis, Electrical Power System

\begin{abstract}
This paper presents parametric design studies of the on-board Electrical Power System (EPS) for a distributed hybrid aircraft propulsion. The work presents the methodology that has been adopted to develop the physics-based models of the EPS components that comprise the electrical machines, power electronic converters, batteries, solid state circuit breakers and power cables. The EPS model is a sizing tool that can be used to evaluate the mass and dimensions of its main components. In addition, it determines the thermal performance of the EPS over six key flight stages of the aircraft mission. The EPS model has been created within a wider multidisciplinary design platform with the aim of sizing the overall aircraft configuration including the aircraft thermal system, the gas turbines and the electrically propelled fans together with the aircraft structure. This paper focusses on the EPS and presents the assessments of three key parameters which are the battery specific energy density, the electric propulsion power and the power split ratio between the generator and the battery. The assessment results demonstrate the significant impact the aforementioned parameters can have on the weight of the battery, the electrical power system and hence the aircraft fuel burn. The work developed within the Clean Sky 2 TRADE project is vital as it develops and applies important physics-based multidisciplinary tools that are required to identify optimum solutions amidst the large number of available conceptual hybrid aircraft architectures.
\end{abstract}

\section{Introduction}

Transport accounts for nearly two thirds of the global crude oil consumption and about a quarter of carbon dioxide (CO2) emissions. Aviation is expected to contribute to around $20 \%$ of greenhouse emissions in the transport sector by 2050 . Important technological advances in the electrification of secondary on-board power for more electric aircraft have been made in recent years bringing significant efficiency improvements [1]. Yet to achieve the challenging ACARE 2020 and Flightpath 2050 goals, bigger step changes may be necessary; that include the adoption of radical disruptive technologies such as the electrification of primary propulsive aircraft power [2]. There are multiple conceptual architectural approaches to hybrid electric aircrafts which are based on a number of expert multidisciplinary technologies. It is of essence that the multidisciplinary aircraft systems be studied not only at the local levels but very importantly at the global aircraft level in relation to each other. That is the only viable way to identify the most sustainable solutions for the novel aircraft architectures for future development.

The Turbo electric Aircraft Design Environment (TRADE) Clean Sky project is developing a design platform that will be used to conduct design and optimisation studies on specific aircraft configurations for a single aisle 190 passengers aircraft. The scope of the platform is to develop advanced structural models, turbo-electric subsystem including gas turbines, electrical power systems, thermal management and cooling systems, and perform design analysis and optimisation studies[3] [4]. This study is based on a particular hybrid electrical distributed propulsion configuration developed by the TRADE consortium. The structural model is being developed by the Technische Universität Berlin (TU Berlin). The gas turbines, thermal system model and mission models are being developed by Mälardalen University (MDH), Sweden in collaboration with Modelon (MOD), Sweden and Germany. The design and optimisation platform is being developed by MOD.

This work presents the methodology that has been adopted to develop physics-based models of the on-board EPS for investigation of the hybrid electrical distributed propulsion configuration. It presents the assessment studies of a few key parameters of the EPS and analyses the results of these studies. The on-board EPS, developed by the University of Nottingham, is a key part of the hybrid electrical aircraft configurations being investigated within TRADE.

The manuscript is organised in six sections. After the introduction, section 2 describes the architecture of the distributed hybrid electric aircraft considered in this work describing the interconnections between the different EPS components. The design methodology adopted to model each of the EPS components is presented in section 3. The EPS 
components include the electrical machine (both motor and generator), the power electronics converter, the solid state circuit breaker, the power cable (both in AC and DC) and the battery. A few concepts regarding hybrid electric aircraft have been used in this paper and is presented in section 4 . The design methodology adopted for the different EPS subcomponents results in highly flexible models suitable for design and optimization studies. Section 5 presents parameters assessments and analyses the results, demonstrating the capabilities of the developed models. Section 6 concludes the paper.

\section{Aircraft System Architecture}

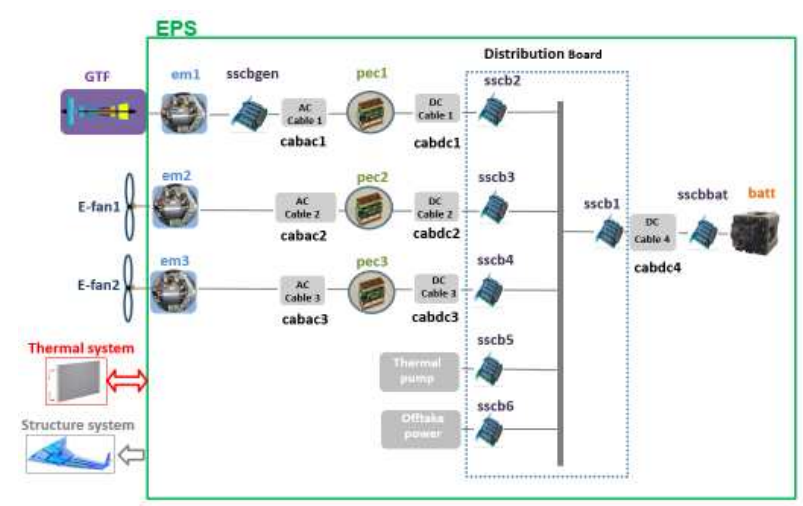

Figure 1: The electrical power system for hybrid electric propulsion considered in this study

The electrical components of the EPS are assumed to be symmetrically distributed on the left and right hand sides of the aircraft. Figure 1 depicts the interconnections of the main electrical components of half of the on-board EPS. Figure 2 that has been developed by TU Berlin within the TRADE project, shows the initial locations of the EPS components on the aircraft. Figure 1 also shows the interconnections between the EPS model and the other system models included in the platform that comprise the gas turbine, the thermal system and the aircraft structure.

Within the EPS, the main electrical loads include two ducted electrical fans (E-fans 1 and 2) for propulsion, one thermal pump for the thermal system and offtake power for feeding onboard electrical loads such as flight controls. These loads receive electrical power from the electrical machine (em1) coupled with the Boosted Gas TurboFan (GTF) as well as from the battery (batt) during the different flight stages. The GTF power is bidirectional, hence the GTF can both supply power to the EPS and receive a power boost from EPS supplied by the battery through em1. The battery can provide power to the EPS and can also be charged during certain flight stages. The electrical machine (em1) can work in both generation and motoring modes. The machine (em1) operates as a generator in this work and is also referred to as (gen). The power electronic converter (pec1) as well as the protective solid state circuit breakers (sscbgen, sscbbat, sscb1 and sscb2) are therefore bidirectional and allow power flow in both directions. The e-fans power is unidirectional, hence em 2 and em3 only operate as motor and they are supplied by pec 2 and pec3, respectively.

DC power is transmitted between the battery and the three power electronic converters (pec1, pec2, pec3) through the DC protective solid state circuit breakers (sscbbat, sscb1- sscb6) and the DC cables (cabdc1 - cabdc4) as shown in Fig. 1. AC power is transmitted from pec 1 and em1 through sscbgen and an ac cable (cabac1). The converters pec 2 and pec3 convert DC power to AC power for the AC electrical machines em2 and em 3 through the AC cables (cabac2, cabac3) respectively. The devices pec2, pec3, sscb3 to sscb6 are unidirectional allowing power to flow in one direction to their respective loads. The distribution board (DB) houses the sscb1 to sscb6.

\section{EPS models sizing and performance calculation}

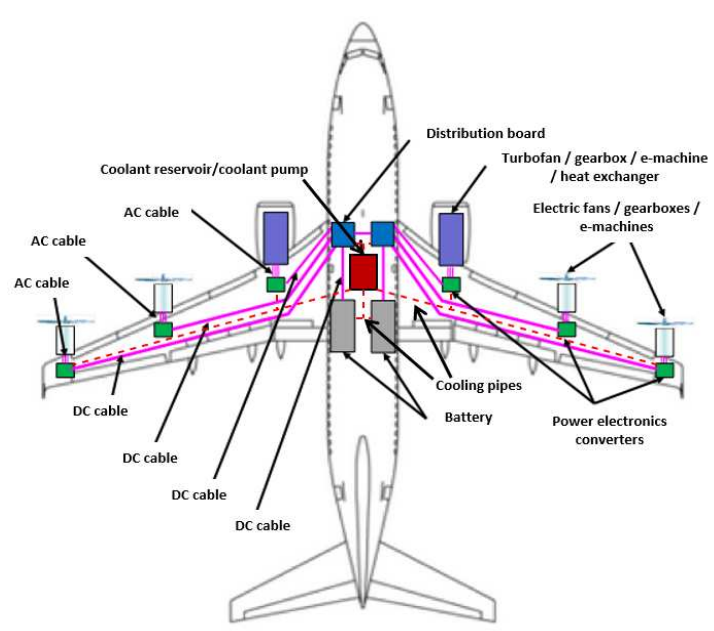

Figure 2: Diagram showing initial locations of the on-board EPS components on the aircraft

The EPS model receives different sets of power and speed profiles from the GTF, E-fan1 and E-fan2, respectively. The power and speed profiles can vary over the six flight stages considered which are taxi, take-off, climb, cruise, approach \& descent and landing. Furthermore, the EPS model receives from the thermal system model the cooling parameters comprising of coolant inlet temperature and flowrate. The EPS model performs the sizing calculation of its different components and returns their respective masses and main dimensions to the aircraft structure model. The EPS model also performs some thermal analysis during the aforementioned flight stages and gives to the thermal system model the heat losses and pressure drops for each EPS component. Within the EPS model, each component calculates a set of output power profile for the next component it is connected to. In this manner, the power requirements are transmitted from one electrical component to the next in the EPS. For instance, once em1, em 2 and em 3 have been sized based on the power and speed profile provided by GTF, E-fan1 and E-fan2 respectively, the models calculate, for the six flight stages, their heat losses and power factors. This information is then employed to compute the output power profile for the next electrical models, which are sscbgen, cabac 2 and cabac 3 for em1, em2 and em3, respectively. The aforementioned "Thermal system" and "Structure system" models of the aircraft, which size the thermal management system of the 
aircraft and the aircraft structure respectively, are not within the scope of this work and will be covered in future publications. The key capability of each of the EPS component model, shown in Figure 1, which are to perform the sizing estimation and performance analysis during key operating points in the mission flight profile will be detailed in the subsequent subsections.

\subsection{Electrical machine}

A Surface Permanent Magnet Synchronous Machine (SPMSM) has been selected as machine topology in this work for the three electrical machines (EMs) considered. The machine is assumed to be intensively cooled by means of oil cooling channels in the slots. SPMSM machines are well known for their greater power density and efficiency compared to different topologies, therefore they are considered good candidates for aerospace applications [5] [6] [7]. The EM model fixed input parameters used in this work are summarized in Table 1.

Table 1: Parameter values for electrical machine

\begin{tabular}{|l|l|}
\hline Parameters & Values \\
\hline Length over diameter aspect ratio $L / D_{s i}$ & 1.5 \\
\hline Inlet temperature of the oil coolant $(\mathrm{K})$ & 373 \\
\hline Mass flowrate of the oil coolant $(\mathrm{kg} / \mathrm{s})$ & 5 \\
\hline
\end{tabular}

Once the mechanical inputs (power and speed profiles) and thermal inputs (flowrate and inlet temperature profiles of the coolant) are received the EM can be sized and the weight and main dimensions returned as outputs to the aircraft structure model. The EM performance are then calculated for the six flight stages and the EM heat losses and pressure drop across the cooling channels are returned to the thermal system model. Furthermore, from the heat losses and power factors during the flight stages, the Volt-Ampere requirements can be calculated and returned to the next electrical model (i.e. sscbgen and cabac 2 and cabac 3 for em1, em 2 and em 3 , respectively).

The EM sizing tool implemented in the model is fully analytical and it works under the assumption of linear magnetic behaviour of the ferromagnetic materials. The EM is designed considering a single operating point, which correspond to the highest power from the input power profile. An additional input, the length over diameter aspect ratio $L / D_{s i}$, is included as a design variable. Indeed, this aspect ratio influences the EM size and weight and can be used to optimize the design. Once the abovementioned inputs have been received a system of two non-linear equations has to be solved as in (1) in order to obtain two important machine quantities: the inner stator diameter $D_{s i}[\mathrm{~m}]$ and the machine peak electrical load $\widehat{K}_{s i}[\mathrm{~A} / \mathrm{mm}]$.

$$
\left\{\begin{array} { l } 
{ \text { eq. } 1 } \\
{ \text { eq. } 2 }
\end{array} \rightarrow \left\{\begin{array}{l}
D_{s i} \\
\widehat{K}_{s i}
\end{array}\right.\right.
$$

"eq. 1" represents the torque equation for a SPMSM [7] while "eq. 2" is the thermal equation [7] which assumes a steady state lumped parameter thermal network. At this conceptual design stage it is assumed that all the copper loss in the slots are extracted through the oil coolant. The implemented sizing model allows then to capture the variation of the EM size not only with the mechanical requirements (power and speed) but also with the coolant properties.

\subsection{Power electronics converter}

A modular approach has been selected to model the pec for two main reasons. First, the basic modules can be arranged in parallel and series in multi-level configuration to cater for the operating voltage and current of the system [8]. Secondly, modularity allows for easier maintenance, faster time to manufacture and assemble, and introduces increased redundancy as and if required. The pec model is constructed around basic $200 \mathrm{~kW}$ and a $500 \mathrm{~kW}$ modules and each module have its own specifications with a temperature limit of $100^{\circ} \mathrm{C}$, and efficiency values between 0.96 and 0.97 as shown in Table 3 . The pec model takes as input the power profile from the previous model it is connected to, and the input temperature and the mass flow rate from the thermal system of the aircraft. The size of the pec modules is computed based on the peak power of the mission profile.

Table 2: Parameter values for power electronic converter modules

\begin{tabular}{|l|l|l|}
\hline $\begin{array}{l}\text { Parameters } \\
\text { (pec modules) }\end{array}$ & $\begin{array}{l}\mathbf{2 0 0} \mathbf{k W} \\
\text { pec module }\end{array}$ & $\begin{array}{l}\mathbf{5 0 0} \mathbf{k W} \\
\text { pec module }\end{array}$ \\
\hline Specific power density (kW/Kg) & 20 & 25 \\
\hline Power density (kW/m3) & 40,000 & 50,000 \\
\hline Maximum temperature limit (K) & 373 & 373 \\
\hline Efficiency at nominal power & 0.96 & 0.97 \\
\hline
\end{tabular}

Cold plates are used to dissipate the power loss from the pec modules, based on the specifications of the standard vacuum brazed cold plate model CP20 from Lytron as given in Table 3 . The thermal component of the pec model determines the output temperature and the output pressure drop of the cooling fluid for the thermal system. It also computes the size of the cold plates.

Table 3: Parameter values for standard cold plates

\begin{tabular}{|l|l|}
\hline Parameters $($ Standard cold plate) & Values \\
\hline Nominal mass flow rate $(\mathrm{kg} / \mathrm{s})$ & 0.047 \\
\hline Mass $(\mathrm{kg})$ & 0.05 \\
\hline Volume $\left(\mathrm{cm}^{3}\right)$ & $51 \times 51 \times 3.3$ \\
\hline Thermal resistance $(\mathrm{K} / \mathrm{W})$ & 0.042 \\
\hline Specific heat capacity of coolant $((\mathrm{J} / \mathrm{KgK})$ & 2000 \\
\hline
\end{tabular}

The mass and volume of the pec model is computed based on computed mass and size of the pec modules, the cold plates as well as the associated controller; these parameters are generated for the structure model.

\subsection{Battery}

The performance of the battery is dependent to a great extent on the material used [9]. The battery model for this configuration is based on the technology of Lithium Ion (LiIon) with a specific energy density (SED) of $0.5 \mathrm{kWh} / \mathrm{kg}$, a power density of $0.9 \mathrm{~kW} / \mathrm{kg}$, and a constant efficiency of $96 \%$, as listed in Table 4. Further, the state of charge (SOC) margin for the battery is assumed as being $20 \%$ but can be varied by the user. The battery model receives as input the required power of the EPS across the different flight stages from the sscbbat and the time durations from a mission model, and determines the total energy requirement over the entire flight 
mission. Designs may require batteries with high energy capacity and/or high peak power. In order to provide the peak power and peak energy, the battery modules are sized based on the maximum values obtained from both specific power density and specific energy density computations. The thermal management of batteries is known to be an important aspect of design since temperature affects both the performance and the battery lifetime. The current battery model uses cold plates that are connected to the thermal system network of the overall aircraft system, as an effective method of heat transfer. The cold plates are modelled based on the approach adopted for the pec cold plates and described in section 3.3 and as per specification given in Table 2 .

Table 4: Parameter values for the battery
\begin{tabular}{|l|l|}
\hline Parameters & Values \\
\hline Specific energy density $(\mathrm{kWh} / \mathrm{Kg})$ & 0.5 \\
\hline Specific power density $(\mathrm{kW} / \mathrm{kg})$ & 1.5 \\
\hline Maximum temperature limit $(\mathrm{K})$ & $293-333$ \\
\hline Efficiency at nominal power & 0.96 \\
\hline Reserve state of charge $(\%)$ & 20 \\
\hline
\end{tabular}

\subsection{Solid state circuit breaker}

There are several types of devices to protect both AC and DC electrical power systems. These include electromechanical devices, hybrid circuit breakers and solid state circuit breakers (sscb). The advantage of sscbs is their shorter operating time and their compact packaging $[10,11]$. The sscb model follows the same modular modelling approach in design as the pec model. Two types of sscbs have been considered in this work namely unidirectional and bidirectional, as per specifications given in Table 5 and Table 6.

Table 5: Parameter values of unidirectional SSCBs
\begin{tabular}{|l|l|l|}
\hline $\begin{array}{l}\text { Parameters } \\
\text { (Unidirectional sscbs ) }\end{array}$ & $\begin{array}{l}\mathbf{2 0 0} \mathbf{~ k W} \\
\text { sscb module }\end{array}$ & $\begin{array}{l}\mathbf{5 0 0} \mathbf{~ k W} \\
\text { sscb module }\end{array}$ \\
\hline $\begin{array}{l}\text { Specific power density } \\
(\mathrm{kW} / \mathrm{Kg})\end{array}$ & 60 & 75 \\
\hline Power density (kW/m3) & 120,000 & 150,000 \\
\hline $\begin{array}{l}\text { Maximum temperature } \\
\text { limit (K) }\end{array}$ & 373 & 373 \\
\hline $\begin{array}{l}\text { Efficiency at nominal } \\
\text { power }\end{array}$ & 0.990 & 0.994 \\
\hline
\end{tabular}

Table 6: Parameter values for the bidirectional SSCBs

\begin{tabular}{|l|l|l|}
\hline $\begin{array}{l}\text { Parameters } \\
\text { (Bidirectional sscbs) }\end{array}$ & $\begin{array}{l}\mathbf{2 0 0} \mathbf{~ k W} \\
\text { sscb module }\end{array}$ & $\begin{array}{l}\mathbf{5 0 0} \mathbf{~ k W} \\
\text { sscb module }\end{array}$ \\
\hline $\begin{array}{l}\text { Specific power density } \\
(\mathrm{kW} / \mathrm{Kg})\end{array}$ & 30 & 38 \\
\hline Power density (kW/m3) & 50,000 & 60,000 \\
\hline $\begin{array}{l}\text { Maximum temperature } \\
\text { limit (K) }\end{array}$ & 373 & 373 \\
\hline $\begin{array}{l}\text { Efficiency at nominal } \\
\text { power }\end{array}$ & 0.990 & 0.994 \\
\hline
\end{tabular}

\subsection{Power cable}

The power cable (ac or dc) model receives as inputs the design points of power over the six flight stages from the electrical component it is connected to. Information on the length of the cable is obtained from the structure model. The power cable then generates the mass per length and cross sectional area of the cable. It is assumed that the cables are naturally cooled.
The cable design is heavily influenced by the voltage level chosen for the EPS. Even though multi-kilovolts are used for terrestrial application, the highest accepted voltage for the aircraft distribution system is $+270 \mathrm{Vdc}$ [10]. Given the above consideration, a voltage level of $600 \mathrm{Vdc}$ has been chosen as the default voltage for the EPS in this study [12]. Yet, it is to be noted that the user is able to vary the dc voltage level. The modelling approach of the ac and dc power cables is based on the works described in [12]. The cable selected is nickel-plated aluminium with $600 \mathrm{~V}$ isolation, designed for an initial temperature of $60{ }^{\circ} \mathrm{C}$ and a final temperature of $125{ }^{\circ} \mathrm{C}$. The cable models use the parameters as given in Table 7 [12].

\begin{tabular}{|l|l|}
\hline Table 7: Parameter values for power cables \\
\hline Parameters & Values \\
\hline Density of a conductor $(\mathrm{kg} / \mathrm{m} 3)$ & 3100 \\
\hline Current density of a conductor $(\mathrm{A} / \mathrm{m} 2)$ & $3.9 \mathrm{e} 6$ \\
\hline Electrical conductivity of a conductor S/m & $32 \mathrm{e} 6$ \\
\hline Nominal DC voltage $(\mathrm{V})$ & 600 \\
\hline Power factor for ac cable & 0.95 \\
\hline
\end{tabular}

\section{Hybridisation concepts}

The power for the two electrical fans varies during the aforementioned six different flight stages. An estimated usage rate ratio $\left(U R R_{i}\right)$ has been applied to the nominal power for the two electrical fans $\left(P_{\text {efan }}\right)$ during each flight stage represented by ' $i$ ' to estimate the corresponding power for the electrical fans for each flight stage $\left(P_{\text {efan_i }}\right)$ as depicted in Figure 3 and shown in Table 8 and equation (2).

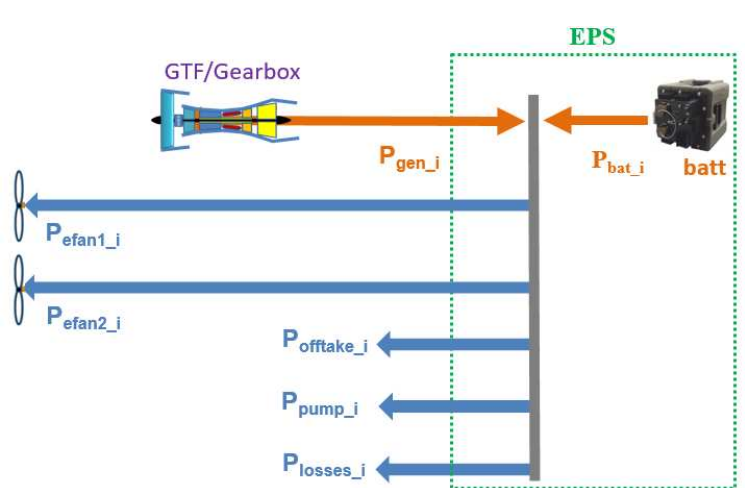

Figure 3: Diagram showing the power flow in the EPS

\begin{tabular}{|l|l|l|l|l|l|l|}
\hline $\begin{array}{l}\text { Flight } \\
\text { Stage }\end{array}$ & $\begin{array}{l}\text { Taxi } \\
\text { In/Out }\end{array}$ & Take off & Climb & Cruise & Descent & $\begin{array}{l}\text { Approac } \\
\text { h/ } \\
\text { Landing }\end{array}$ \\
\hline $\mathrm{i}$ & 1 & 2 & 3 & 4 & 5 & 6 \\
\hline$U R R_{i}$ & 0.02 & 1.0 & 0.75 & 0.5 & 0.2 & 0.2 \\
\hline
\end{tabular}

$$
P_{\text {efan } \_}=U R R_{i} * P_{\text {efan }}
$$

The power $\left(P_{\text {efan_i } i}\right)$ is generated equally from the electrically propelled fan $1\left(P_{\text {efan } \__{i}}\right)$ and fan $2\left(P_{\text {efan }{ }_{-} i}\right)$ :

$$
P_{\text {efan }}=P_{\text {efan } 1_{i}}+P_{\text {efan } 2_{i}} ; \quad P_{\text {efan } 1_{-} i}=P_{\text {efan } 2_{i}}
$$


The electrical loads include the electrical offtake power $\left(P_{\text {offtake }} i\right)$, the electrical power for the thermal pump $\left(P_{\text {pump }} i\right)$. The usage rate ratios given in Table 8 have been applied to the nominal values of $P_{\text {offtake }}$ and $P_{\text {pump }}$ to obtain the corresponding values for each flight stage for convenience. The total electrical load for half of the aircraft $P_{L H A_{-} i}$ is given by the sum of $P_{\text {efan } 1_{-} i}, P_{\text {efan } 2_{-} i}, P_{\text {offtake } \_} i$ and $P_{\text {pump } \_} i$ :

$$
P_{\text {LHA }_{-} i}=P_{{\text {efan } 1_{\_} i}}+P_{\text {efan }_{i}}+P_{\text {offtake } \__{-} i}+P_{\text {pump }_{-} i}
$$

The total electrical load $\left(P_{L H A_{-} i}\right)$ in addition to the power losses during each flight stage $\left(P_{\text {losses }_{-} i}\right)$ is supplied by both the gas turbine generator $\left(P_{g_{e n} i}\right)$ and the battery $\left(P_{\text {bat } i}\right)$ :

$$
P_{\text {gen } \_i}+P_{\text {bat } \_i}=P_{\text {LHA } \_} i+P_{\text {losses } \_i}
$$

The term power split $(P S)$ in this study refers to the ratio of the battery power usage over the total electrical power required for half of the aircraft as given below:

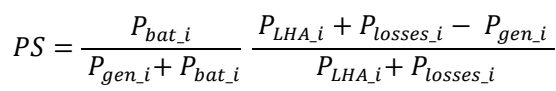

Of note is that since the EPS power losses are not known at the outset and can only be determined by running the EPS simulation model. An estimated power split $P S_{\text {est }}$ excluding power losses has been used as a way to determine the power that is requested from the generator $\mathrm{P}_{\text {gen_i }_{\mathrm{i}}}$ :

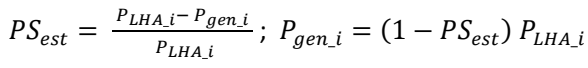

The power required for the generator can be determined from equation (7) and fed into the simulation model of the EPS. The power losses $\left(P_{\text {losses_i }}\right)$ of the EPS and the power required from the battery $\left(P_{\text {bat } \_}\right)$are determined by running the simulation model.

\section{Parametric Investigations}

This section presents an example of the parametric study that can be performed with the presented EPS model. The variations of SED, $P_{\text {efan }}$ and $\mathrm{PS}_{\text {est }}$ have been considered as three different case studies. The main parameter values that have

\begin{tabular}{|c|c|}
\hline Parameters & Values \\
\hline Nominal power for two electrical fans for aircraft $(\mathrm{kW})$ & 2000 \\
\hline Nominal offtake power $(\mathrm{kW})$ & 150 \\
\hline Nominal power for thermal pump (kW) & 100 \\
\hline Mass flow rate of coolant from thermal system $(\mathrm{kg} / \mathrm{s})$ & 5 \\
\hline Inlet temperature of coolant from thermal system (K) & 323 \\
\hline Length of dc cable 1 & 10 \\
\hline Length of dc cable 2 & 26 \\
\hline Length of dc cable 3 & 30 \\
\hline Length of dc cable 4 & 0.3 \\
\hline Length of ac cables $1,2,3$ & 0.3 \\
\hline Duration of taxi out and taxi in & 12 \\
\hline Duration of take-off (min) & 2 \\
\hline Duration of climb (min) & 13 \\
\hline Duration of cruise (min) & 22 \\
\hline
\end{tabular}
been used in this study are given in Tables 1 to 8 and Table 9 and are based on the studies being undertaken within the TRADE project in collaboration with MDH, TUB and MOD.

\begin{tabular}{|l|l|}
\hline Duration of descent (min) & 37 \\
\hline Duration of approach and landing (min) & 6 \\
\hline
\end{tabular}

\subsection{Battery specific energy density}

One of the technical challenges for hybrid electric aircraft is low batteries SED. The influence of the battery SED on the mass of the battery $M_{\text {batt }}$ and mass of the EPS $M_{E P S}$ has been investigated in this case study by varying the SED from current technology level $(0.2 \mathrm{kWh} / \mathrm{kg})$ to future technology level $(1.2$ $\mathrm{kWh} / \mathrm{Kg})$. The values for $P_{\text {efan }}$ and $P S_{\text {est }}$ have been fixed to $2000 \mathrm{~kW}$ and 0.05 respectively. The results are depicted Error! Reference source not found..

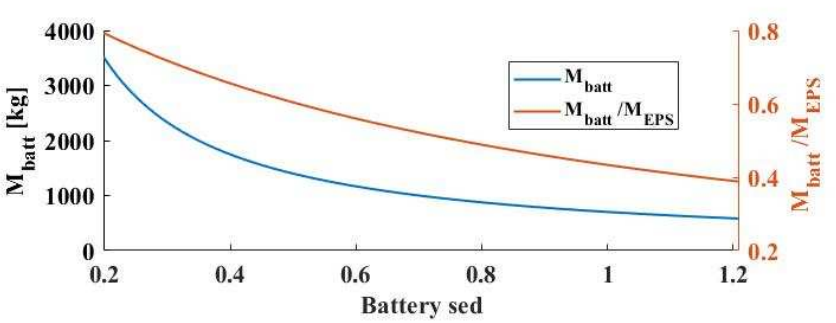

Figure 4: Battery mass and ratio of battery mass to EPS mass as a function of battery specific energy density (SED)

As expected, the battery mass $\mathbf{M}_{\text {batt }}$ is significantly reduced when SED for future technologies is considered. Indeed, $\mathbf{M}_{\text {batt }}$ is reduced from around $3500 \mathrm{~kg}$ to nearly $600 \mathrm{~kg}$. The chart in Error! Reference source not found. also displays the trend of the battery mass to EPS mass ratio $\left(\mathrm{M}_{\text {batt }} / \mathrm{M}_{\mathrm{EPS}}\right)$ as a function of SED. It can be noticed that with the current battery technology level the battery mass would represent almost 80 $\%$ of the mass of the on-board EPS considered in this study. This figure is considerably reduced with the battery mass to EPS mass ratio being equal to around $40 \%$ when the battery $\mathrm{SED}$ is equal to $1.2 \mathrm{kWh} / \mathrm{Kg}$ as anticipated for future technologies.

\subsection{Power for electric propulsion}

This subsection investigates the impact of the electrical fans power $P_{\text {efan }}$ on the battery and generator mass. The values of $P S_{\text {est }}$ and battery SED are kept constant at 0.05 and 0.5 $\mathrm{kWh} / \mathrm{Kg}$ respectively. The $P_{\text {efan }}$ is then increased from $200 \mathrm{~kW}$ to $2000 \mathrm{~kW}$ and the influence on the mass of the two electrical 
power sources, the battery and the generator is presented in Figure 5.

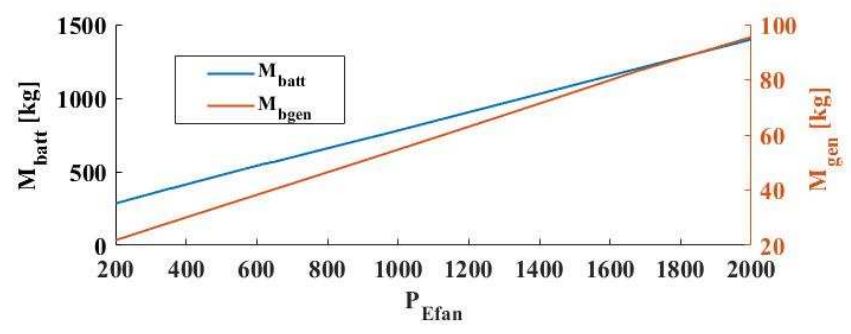

Figure 5:Battery and generator mass as function of electrical fan power $\left(P_{\text {efan }}\right)$

It can be observed that both the battery and the generator masses increase as they are required to provide more power to the electric fans. However, it is straightforward to notice that with the considered battery SED, the battery mass increase is significantly larger than the one of the generator.

\subsection{Power split between Generator and Battery}

In this study, the proportion of power to be provided from the generator for the electrical loads is determined by the user setting the $P S_{\text {est }}$ as per equation (7). This case study assesses the impact of the $P S_{\text {est }}$ on the battery and generator masses. The values of the $P_{\text {efan }}$ and battery SED are kept constant at 2000 $\mathrm{kW}$ and $0.5 \mathrm{kWh} / \mathrm{Kg}$, respectively. As can be observed from Figure 6 the $P S_{\text {est }}$ ratio increases from 0.01 to 0.5 leading on one side to a reduction of the generator mass and on the other to an increment of the battery mass. Again, the increase of the battery mass is significantly larger than the decrease of the generator mass.

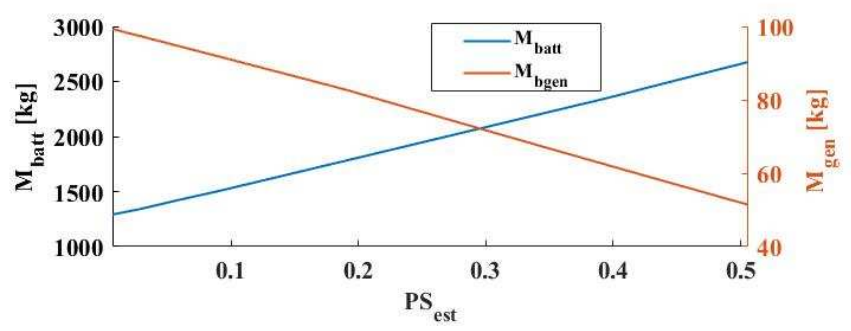

Figure 6 Battery mass and generator mass as a function of power split ratio between battery and generator $P S_{e s t}$

\section{Conclusion}

This work presents physics-based modelling approaches of the on-board EPS for one particular configuration of a distributed hybrid electric propulsion chain that includes electrical machines, power electric converters, battery, solid state circuit breakers and power cables. The three assessments presented in this paper show that the battery specific energy density, the power of the electrical fans and the power split ratio between the generator and the battery have significant impact on the masses of the two main electrical power sources, the battery and the generator, and therefore on the aircraft fuel efficiency. The benefits of the physics-based EPS models presented in this work is that it offers the possibility of a wide number of investigations to be carried out to determine the optimum solutions that can serve the advancement of hybrid technologies for aircraft. Future works will extend the present study to demonstrate how the EPS components impact other systems of the aircraft such as the thermal system, the gas turbine, the mission model, the aircraft structure design, and in addition will present results from optimisation studies at the aircraft level aimed to minimise the block fuel consumption. The work developed within the TRADE project is vital as it develops and applies important physics-based multidisciplinary tools that are required to identify optimum solutions amidst the large number of available conceptual hybrid aircraft architectures.

\section{Acknowledgements}

This project has received funding from the Clean Sky 2 Joint Undertaking under the European Union's Horizon 2020 research and innovation programme under grant agreement number 755458 .

The authors would like to acknowledge the contributions of the TRADE project team: Xin Zhao, Smruti Sahoo and Konstantinos Kyprianidis from Mälardalen University, Sweden; Claire Jacob, Annika Scheunemann, Tomasz Pronobis and Andreas Bardenhagen from Technische Universität Berlin, Germany; Jonatan Rantzer, Clément Coïc, Katrin Prölss, Edward Ekstedt, Jim Claesson and Michael Sielemann from Modelon, Sweden and Germany; Wolf Krüger and Tobias Hecken from the German Aerospace Center DLR.

\section{References}

[1] K. Ni et al., "Electrical and Electronic Technologies in MoreElectric Aircraft: A Review," IEEE Access, vol. 7, pp. 76145-76166, 2019.

[2] A. Krein and G. Williams, "Flightpath 2050: Europe's vision for aeronautics," Innovation for Sustainable Aviation in a Global Environment: Proceedings of the Sixth European Aeronautics Days, Madrid, vol. 30, 2012.

X. Zhao et al., "A Framework for Optimization of Hybrid Aircraft," in ASME Turbo Expo 2019: Turbomachinery Technical Conference and Exposition, 2019, vol. Volume 3: Coal, Biomass, Hydrogen, and Alternative Fuels; Cycle Innovations; Electric Power; Industrial and Cogeneration; Organic Rankine Cycle Power Systems, V003T06A012.

X. Zhao, S. Sahoo, K. Kyprianidis, J. Rantzer, and M. Sielemann, "Off-design performance analysis of hybridised aircraft gas turbine," The Aeronautical Journal, vol. 123, no. 1270, pp. 19992018, 2019.

W. Cao, B. C. Mecrow, G. J. Atkinson, J. W. Bennett, and D. J. Atkinson, "Overview of electric motor technologies used for more electric aircraft (MEA)," IEEE transactions on industrial electronics, vol. 59, no. 9, pp. 3523-3531, 2011.

[6] D. Golovanov, L. Papini, D. Gerada, Z. Xu, and C. Gerada, "Multidomain Optimization of High-Power-Density PM Electrical Machines for System Architecture Selection," IEEE Transactions on Industrial Electronics, vol. 65, no. 7, pp. 5302-5312, 2017.

[7] J. Pyrhonen, T. Jokinen, and V. Hrabovcova, Design of rotating electrical machines. John Wiley \& Sons, 2013.

[8] C. Gammeter, F. Krismer, and J. W. Kolar, "Weight and efficiency analysis of switched circuit topologies for modular power electronics in MEA," IECON 2016 - 42nd Annual Conference of the IEEE Industrial Electronics Society, Florence, 2016, pp. 36403647. 
[9] J. Hoelzen et al., "Conceptual design of operation strategies for hybrid electric aircraft," Energies 2018, volume 11, issue 1.

[10] C.Jones, P.J.Norman, S.J.Galloway, M.Armstrong, and A.Bollman, "Comparison of candidate architectures for future distributed propulsion aircraft," IEEE transactions on applied superconductivity, vol 23, Sep 2016

[11] C. Pornet and A. T. Isikveren, "Conceptual design of hybridelectric transport aircraft," Progress in Aerospace Sciences, vol. 79, pp. 114-135, 2015.

[12] S. Teichel et al., "Design considerations for the components of electrically powered active high-lift systems in civil aircraft," CEAS Aeronautical Journal, vol. 6, no. 1, pp. 49-67, 2015. 